\title{
Changes in same-different laterality patterns as a function of practice and stimulus quality
}

\author{
JOSEPH B. HELLIGE \\ University of Southern California, Los Angeles, California 90007
}

\begin{abstract}
Accuracy and reaction time (RT) of judgments about sameness vs. difference of (a) names of two letters and (b) shapes of two nonverbal forms were examined for stimuli presented to the center, left (LVF), and right (RVF) visual fields. For same-name letter pairs during Experiment I, responses were more accurate and faster for LVF than for RVF trials on an initial 90-trial block, but this difference was reversed by a third 90 -trial block. The RVF advantage for RT was maintained over Trial Blocks 4 and 5, given during a second session, but had disappeared on Trial Blocks 6 through 9 as RT reached the same asymptotic level for both visual fields. No LVF-RVF differences were obtained at any level of practice for different-name letter pairs or for any of the form pairs. Experiment II replicated the shift from LVF toward RVF advantage that occurred over the first three trial blocks of Experiment I and demonstrated that such a shift does not occur when the letters are perceptually degraded. The results were discussed in terms of differences in cerebral hemisphere specialization for visuospatial vs. abstract stages of letter processing and changes with practice in the relative difficulty of these stages.
\end{abstract}

Because stimuli from the left and right visual fields are projected directly to the contralateral cerebral hemisphere, visual laterality patterns have come to be taken as indicators of relatively permanent information processing differences between the left and right cerebral hemispheres (e.g., Kimura, 1966, 1973). One important paradigm for studying some of these information processing specializations involves judgments about whether two simultaneously presented letters have the same name. To insure that some amount of verbal processing is required to perform the task, the two letters typically differ in case, so that responding on the basis of a physical match is impossible. In a typical experiment of this type, a pair of letters is presented on each trial to the left visual field (LVF) or to the right visual field (RVF) and the subject presses one key if the letters have the same name and another key if they do not. The task is designed to produce few errors, and subjects are instructed to respond as quickly as possible. Therefore, primary emphasis has been on the reaction time (RT) of correct responses.

The prototypical finding usually cited for this paradigm is that RTs are faster on RVF-left hemisphere (RVF-LH) trials than on LVF-right hemisphere (LVF-RH) trials, and the conclusion usually reached is that the left cerebral hemisphere is specialized for verbal information processing (e.g., Geffen, Bradshaw, \& Nettleton, 1972). How-

This research was supported in part by a grant to the author from the National Institute of Mental Health (MH27116-01) and in part by Biomedical Sciences Support Grant RR07012-09. Requests for reprints should be sent to Joseph B. Hellige, Psychology Department, University of Southern California, University Park, Los Angeles, California 90007. ever, there remain some exceptions to this prototypical result. The majority of studies that report an RVF-LH RT advantage for letter pairs with the same name find no such advantage for letter pairs of different names (e.g., Cohen, 1972; Davis \& Schmit, 1973; Geffen et al., 1972, in the mixed condition), despite the fact that both types of trials would seem to demand verbal processing. In addition, several recent experiments that have used this paradigm have found either no visual field difference in RT for any of the letter pairs (Wilkins \& Stewart, 1974) or have found an LVF-RH advantage for such pairs (Hellige, 1975, Experiment 2; Lefton \& Haber, 1974, Experiment 3). If paradigms such as these reveal anything about the specialization of the cerebral hemispheres, it is important to discover some of the reasons for these discrepant findings.

A review of the methods used in this general letterpair paradigm suggests that level of practice may be an important determiner of the observed laterality patterns. Those studies that have reported faster RTs on RVF-LH trials have used more practiced subjects and analyzed a larger number of experimental trials than have those studies reporting either no visual field difference or faster RTs on LVF-RH trials. If visual laterality patterns reflect only invariant structural properties of the human nervous system, then one would not expect the pattern of results to change systematically with such things as the level of practice. Therefore, it is important to precisely determine the nature of such effects.

In a brief footnote, Hellige (1975) mentions the results of a preliminary investigation of this practice effect. Subjects were given three blocks of 90 trials each, using pairs of different-case letters projected to 
the center, left, or right visual field on each trial. For same-name letter pairs, the RTs on the first block of 90 trials were faster on LVF-RH trials. However, on the second and third blocks of 90 trials, RTs were 26 and $41 \mathrm{msec}$, respectively, faster on RVF-LH trials than on LVF-RH trials. Such results demand a more detailed presentation and additional investigation, especially in light of recently reported changes in auditory laterality patterns as a function of practice (Kallman \& Corballis, 1975). Accordingly, Experiment I was designed to examine both same-different letter-name judgments and same-different nonverbal shape judgments over a period of 3 days with three 90 -trial blocks on each day. The first day allows a detailed replication of the result alluded to by Hellige (1975), while the remaining days allow any practice effects to be examined more thoroughly. The shape judgment task is included to determine whether any practice effects that occur for letter pairs result from the necessity for verbal processing or whether the effects are related to visuospatial processing.

\section{EXPERIMENT I}

\section{Method}

Apparatus. The subject sat at a table facing a $24 \times 20 \mathrm{~cm}$ dark gray screen approximately $75 \mathrm{~cm}$ away at eye level. At the exact center of the screen was a white circular dot which subtended about $0.2^{\circ}$ of visual angle. The dot was present at all times during the experiment. On top of the table were two telegraph keys $30 \mathrm{~cm}$ from each other and equidistant from the center of the table. Above one key was a card with the label SAME, and above the other key was a card with the label DIFFERENT. Stereo headphones (SONY DR 7A) and a Heathkit sine-square generator (MODEL AG-10) were used to present a $560-\mathrm{Hz}$ square wave ready signal at an intensity of approximately $60 \mathrm{~dB}$ re $20 \mathrm{~N} / \mathrm{m}^{2}$. A Kodak Carousel 850 slide projector and a Gerbrands G1166 shutter were used to rear-project visual stimuli onto the stimulus display screen. Response latency and correctness were recorded manually by the experimenter using an Automated Data Systems Model 1248B digital timer.

Stimulus materials. The letters A, B, D, E, G, H, R, T, N, and $Q$, in both upper- and lowercase form (ParaTipe 11204), served as verbal stimuli. The two letters of a pair were arranged one above the other to minimize horizontal scanning effects. When projected, the stimuli appeared as white letters, with an average illumination of approximately $90 \mathrm{~mL}\left(308.4 \mathrm{~cd} / \mathrm{m}^{2}\right)$, on a dark gray background, with an average illumination of $.5 \mathrm{~mL}$ $\left(1.71 \mathrm{~cd} / \mathrm{m}^{2}\right)$. When projected on the screen, each letter subtended between $1.0^{\circ}$ and $0.6^{\circ}$ of visual angle horizontally and between $1.3^{\circ}$ and $0.8^{\circ}$ of visual angle vertically. There was between $0.8^{\circ}$ and $0.6^{\circ}$ of visual angle between the two letters of a pair. Pairs presented centrally were located so that the fixation dot appeared between the two letters and so that the center of each letter pair was lined up with the middle of the screen; i.e., such pairs were centered horizontally and vertically. Pairs presented to the left or right of the fixation dot were in the same vertical position as the central pairs, but the center of the projected letter pairs averaged $3.9^{\circ}$ of visual angle to the left or right of fixation with a standard deviation of $14^{\circ}$

Three stimulus sets were constructed, each containing 15 Same and 15 Different pairs selected randomly from the population of pairs defined by the 10 letters. Each of the 30 stimuli in a set appeared once in each of three visual field locations: centrally, to the right of center, and to the left of center. There were, therefore, six types of letter trials determined by two levels of correct response (Same or Different) and three levels of visual field stimulated. Each sequence contained 15 trials of each type, for a total of 90 trials. The order of presentation of the trial types was random, with the restriction that eati! of tis six tral types appeared three times in each block of 18 trials.

Ten of the irregular polygons scaled by Vanderplas and Garvin (1959) served as nonverbal stimulus forms. The 10 forms used were Nos. 6-26, 6-28, 6-30, 8-25, 8-26, 8-27, 8-29, 8-30, 12-25, and 12-27. Irregular polygons were arranged one above the other and projected in the same way as the letters. When projected, they appeared as solid white forms with an average illumination the same as that for letters. When projected, the forms subtended between $1.4^{\circ}$ and $1.0^{\circ}$ of visual angle horizontally and between $1.2^{\circ}$ and $0.8^{\circ}$ of visual angle vertically. There was between $1.1^{\circ}$ and $1.2^{\circ}$ of visual angle between the stimuli of a pair. All pairs were located in the appropriate location on the screen in the same way as the letters. Three stimulus sets were constructed, each containing 15 Same and 15 Different pairs selected randomly from the population of pairs defined by the pool of forms. Each of the 30 stimuli in a set appeared once in each of the three locations, and order of trial types was determined the same way as for letters.

Procedure. Each subject in the experiment performed either the letter task or the form task and received the three 90-trial sequences of a given stimulus type in a different random order during each of three experimental sessions. The sessions were separated from each other by $48 \mathrm{~h}$. In addition, half of the subjects indicated Same responses with their right hands and Different responses with their left hands, while the remaining subjects had the opposite arrangement.

At the beginning of the experiment, subjects were instructed to wear the headphones at all times and keep the first finger of the right hand on the right telegraph key and the first finger of the left hand on the left telegraph key throughout the experimental session. The subjects were told to fixate their gaze on the central fixation dot when the warning tone sounded and maintain that fixation until after they responded. The subjects were then told that on each trial a letter (form) pair would appear on the screen in the center or off to one side. Each subject was also informed that the three locations would be tested in a random order so that the best strategy was to fixate on the central dot whenever the tone sounded. For letters, the subject was told that the letters would always be of different cases and that the task was to press, as quickly as possible, the key labeled SAME if the letters had the same name and the key labeled DIFFERENT if the letter names were different. For forms, subjects were told to press the key labeled SAME if the shapes were the same and the key labeled DIFFERENT if they were different. Both response speed and accuracy were stressed by telling the subject to respond as quickly and as accurately as possible.

Prior to the experimental task during each session, the subject was given 18 practice trials, three of each of the six trial types. After the practice trials for the first session, the subject was given a chance to ask questions about the procedure. The importance of fixating appropriately and of responding as quickly as possible was stressed after each block of 90 trials.

On each trial, a $500-\mathrm{msec}$ warning tone was presented $1.5 \mathrm{sec}$ before the stimulus pair. This interval was chosen to allow time for fixation without demanding that the fixation be maintained for a long period of time. Each stimulus pair was presented for $30 \mathrm{msec}$. The intertrial interval was $6 \mathrm{sec}$ measured from tone onset on Trial $\mathbf{n}$ to tone onset on Trial $\mathbf{n}+1$.

Subjects. Subjects were 12 men and 12 women student volunteers from introductory psychology courses at the University of Southern California. All subjects had normal or corrected-to-normal visual acuity in both eyes, were native speakers of English, were righthanded, and reported fixating on the center of the viewing screen 
at the appropriate times. Subjects were assigned to groups randomly until it became necessary to balance for sex.

\section{Results and Discussion}

Although subjects were counterbalanced for the hand making Same responses and equal numbers of men and women participated, the following analyses are collapsed over these variables. The only significant effect involving either variable was that subjects responded faster if the right hand responded Same and the left hand responded Different than vice versa, $\mathrm{F}(1,20)=5.932, \mathrm{p}<.05$.

Errors. The error rates were generally low, $6.96 \%$ overall. In addition, averaged over letters and forms, there were fewer errors on center trials $(4.3 \%)$ than on peripheral visual field trials and there were about the same number of errors on both LVF (8.23\%) and RVF $(8.22 \%)$ trials. This pattern of results was supported statistically as a large visual field difference with the center trials included, $F(2,44)=26.510$, $\mathrm{p}<.001$, and a nonsignificant visual field difference with the center trials excluded, $F<1$. The lower error rate on center trials indicates that subjects were fixating in the center of the field as instructed so that the center trials were perceived foveally.

On Day 1, there were two additional error rate effects. More errors were made on Same trials $(10.68 \%)$ than on Different trials $(6.9 \%), F(1,20)=$ $6.631, \mathrm{p}<.025$. More importantly, for same-name letter pairs, the percentages of errors on LVF-RH and RVF-LH trials, respectively, were $18 \%$ and $25 \%$ on Trial Block $1,11 \%$ and $10 \%$ on Trial Block 2, and $11 \%$ and $7.2 \%$ on Trial Block 3. Note that the decline in error rate over the three trial blocks was larger for RVF-LH trials than for LVF-RH trials, resulting in a significant Linear Trial Block by Visual Field interaction, $F(1,11)=9.514, p<.025$, and producing a shift away from an initial LVF-RH recognition advantage. No additional error rate effects were significant.

Reaction time. For each response, the time from the onset of the stimulus to the onset of the response was measured. For each of the 90 -trial blocks given to each subject, the median RT of correct responses was determined for each type of trial defined by Same vs. Different and visual field location. For convenience of exposition, the results on center trials will first be contrasted with the results on peripheral location trials followed by a direct comparison of the left and right visual fields.

Table 1 shows the means of the median RTs in milliseconds for Same and Different trials in each visual field location on each of the experimental days. The RTs shown are collapsed over letters and forms and the three 90-trial blocks of each day because the relationship of center to peripheral trials was not influenced by these variables. As Table 1 shows, RTs were faster for center trials than for
Table 1

Mean of Median Reaction Time (Milliseconds) for Each Visual Field Location on Each Day

\begin{tabular}{lllllll} 
& \multicolumn{4}{c}{ Visual Field Location } \\
\cline { 2 - 6 } & \multicolumn{2}{c}{ Center } & \multicolumn{2}{c}{ Left } & \multicolumn{2}{c}{ Right } \\
& Same & Different & Same Different & Same Different \\
\hline Day 1 & 741 & 789 & 838 & 841 & 832 & 832 \\
Day 2 & 676 & 718 & 740 & 742 & 730 & 742 \\
Day 3 & 618 & 674 & 700 & 695 & 688 & 700 \\
\hline
\end{tabular}

either LVF or RVF trials, while RTs did not differ for LVF vs. RVF trials. These results were supported statistically as a significant visual field effect with center trials included, $F(2,44)=37.146, p<.001$, and a nonsignificant visual field effect without center trials, $\mathrm{F}<1$. Table 1 also shows an interesting Center-Left-Right Visual Field by Same-Different interaction that is consistent across all 3 days. On center trials, RTs were faster to Same pairs than to Different pairs while this difference was absent on both left and right visual field trials. Accordingly, there was a significant Visual Field by Same-Different interaction with the center trials included, $F(2,44)=$ $12.403, \mathrm{p}<.001$, but not with the center trials omitted, $F(1,22)=1.323, p>.20$. This Visual Field by Same-Different interaction is consistent with the previous reports of Hellige (1975) and Lefton and Haber (1974), who found a shift away from faster RTs on Same trials as the stimuli were moved away from the fovea on either side.

Figure 1 shows RT in milliseconds as a function of 90-trial blocks (three blocks per day). As can be seen from Figure 1, RTs were faster to form pairs than to letter pairs, $F(1,22)=5.764, p<.025$. This result is consistent with the fact that processing of letter pairs requires a verbal processing stage while processing of form pairs does not, but it may also reflect a difference in the ease of perceptual processing of the two kinds of stimuli. Averaged over visual fields and stimulus type, RTs decreased markedly over the three trial blocks of Day 1 and then decreased at a slower rate over the remaining trial blocks. This pattern produced significant linear and quadratic trends over the nine blocks of trials: Linear $F(1,22)=$ 37.793, $\mathrm{p}<.001$; Quadratic $\mathrm{F}(1,22)=9.950$, $\mathrm{p}<.005$. $^{1}$

Further inspection of Figure 1 indicates that, for same-name letter pairs, RT on Trial Block 1 was slightly faster on LVF-RH trials than on RVF-LH trials, but responses had become $86 \mathrm{msec}$ faster on RVF-LH trials than on LVF-RH trials by Trial Block 3. That is, over Trial Blocks 1 to 3, RT to Same-name letter pairs started out higher and decreased more rapidly on RVF-LH trials than on LVF-RH trials. In addition, the RVF-LH R'T reached asymptote by Trial Block 4 while the LVF-RH RT did not reach the same asymptote until Trial Block 6 . 


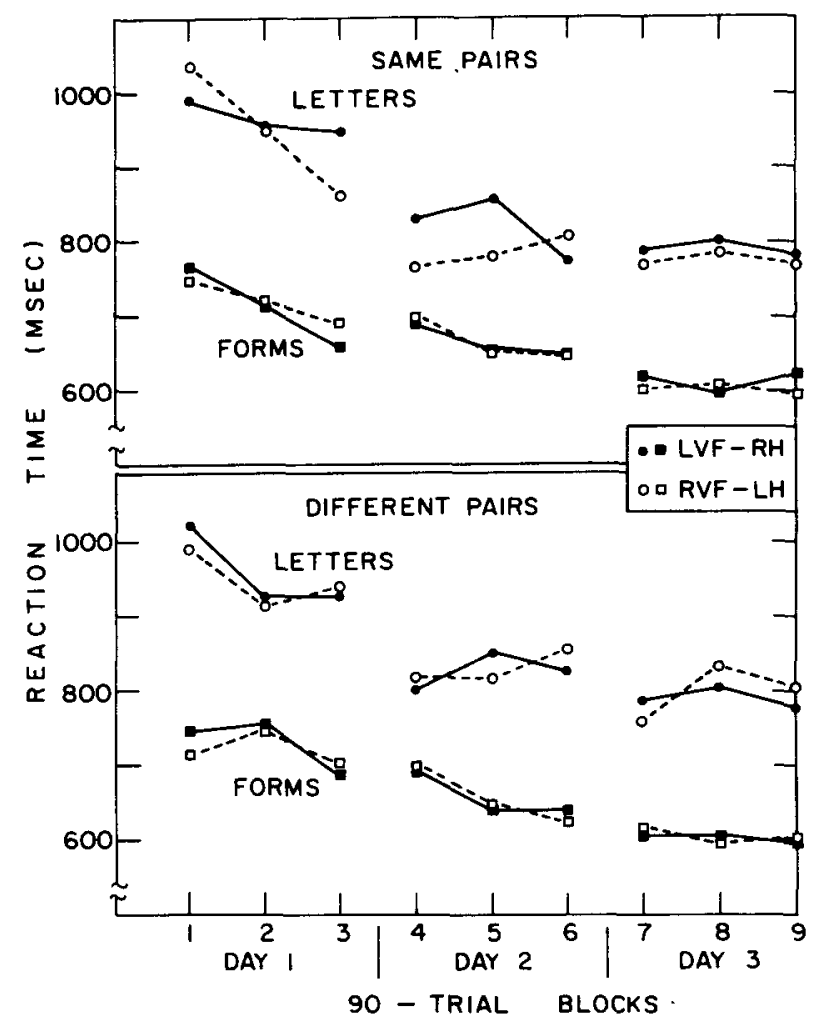

Figure 1. Mean RT in milliseconds as a function of 90 -trial blocks (three blocks per day). The upper and lower panels show RT to Same and Different pairs, respectively. In each panel, the RTs are shown for left-visual-field/right-hemisphere (LVF-RH) and right-visual-field/left-hemisphere (RVF-LH) trials for letters and forms separately.

These different trial block trends produced a significant Quadratic Trial Block by Visual Field interaction for same-name letter pairs, $F(1,11)=6.874$, $\mathrm{p}<.025$. In addition, over the trial blocks of Day 1, 10 of the 12 subjects showed a shift away from the initial LVF-RH advantage (sign test, $p<.05$ ) consistent with the error pattern noted earlier. As suggested by Figure 1, there were no significant effects involving visual field for different-name letter pairs or any of the form pairs.

The results of the present experiment indicate that laterality effects can change quantitatively and qualitatively as the level of practice is varied (cf. Hellige, 1975; Kallman \& Corballis, 1975). For same-name letter pairs, the prototypical RVF-LH advantage was obtained only for levels of practice used in those previous studies reporting such effects (e.g., Cohen, 1972; Davis \& Schmit, 1973; Geffen et al., 1972). The absence or reversal of the prototypical laterality pattern with less practice replicated the results from earlier studies employing corresponding levels of practice (e.g., Hellige, 1975; Lefton \& Haber, 1974; Wilkins \& Stewart, 1974). Therefore, differences in the level of practice may indeed account for much of the discrepancy among earlier studies using these types of stimuli. The present results also indicate that with sufficient practice, considerably more than is common in laterality experiments, RT can eventually reach the same asymptotic level for both RVF-LH and LVF-RH trials under experimental conditions that produce visual field differences at earlier levels of practice. This may occur because the right hemisphere eventually becomes as efficient as the left at handling the relatively simple verbal analysis or because the right hemisphere becomes more efficient at initially encoding the visuospatial information to be transferred via the corpus callosum to the left hemisphere for verbal analysis (e.g., Geffen et al., 1972; Hellige \& Cox, 1976; Kimura, 1966, 1973).

Perhaps the level of practice interacts with hemispheric advantages over early trial blocks because the relative difficulty of visuospatial and abstract-verbal processes is important in determining hemispheric advantage. For adults, the assignment of letter names should be fairly automatic once the relevant visual features have been identified. However, at the beginning of the experiment, the more perceptual, visuospatial aspects of processing the letter pairs may be relatively difficult because subjects are not familiar with the specific type font to be employed, the specific letters to be used, or the specific locations to be tested, and must adjust to the brief stimulus duration. It has been frequently suggested that the right cerebral hemisphere is specialized for visuospatial analysis (e.g., Hellige \& Cox, 1976; Kimura \& Durnford, 1974). Therefore, if the visuospatial aspects of processing are relatively difficult, the typical RVF-LH advantage may be obscured or even reversed at early levels of practice. After a certain amount of practice, the perceptual analysis may become relatively easy and automatic so that it is the abstract, verbal aspects of the task that are relatively more difficult and a shift towards an RVF-LH advantage occurs for both errors and RT. This explanation implies that the general decrease in errors and RT over the early trial blocks results primarily from increasing ease of visuospatial aspects of processing. Consistent with this, responses to form pairs also became more accurate and faster as the experiment progressed. However, for the form pairs, there was no significant shift towards an RVF-LH advantage because, unlike the letters, no verbal processing stage was required.

If the shift over early trial blocks from right toward left hemisphere advantage occurred for samename letter pairs because aspects of visuospatial processing were becoming easier, then it should be possible to prevent such a shift by maintaining the perceptual difficulty of the task. One way to accomplish this would be to present perceptually 
degraded stimuli in a task similar to that employed in Experiment I. Accordingly, two groups of subjects in Experiment II performed the letter-pair task used in Experiment I under different conditions of stimulus quality. Viewing conditions for one group of subjects (intact group) were essentially identical to those of Experiment 1 , while for the other group (degraded group) a grid of dark lines was placed over the viewing screen to increase and maintain the perceptual difficulty of the task. Note that such a manipulation would not be expected to change the difficulty of more abstract, verbal stages of letter naming and comparison but should influence only the initial visuospatial processing stages (see Sternberg, 1969,1975 ). The foregoing explanation of the early Trial Block by Visual Field interaction for samename letter pairs predicts that such an interaction should occur only for the intact group of Experiment II.

\section{EXPERIMENT II}

\section{Method}

The apparatus, stimulus materials, and procedure were identical with those described for Experiment I except that the stimulus duration on each trial was increased to $100 \mathrm{msec}$ for all subjects. In addition, each subject performed only one session-identical to Day 1 of Experiment I-and only the letterpair stimuli were used. One group of subjects, the intact group, received stimuli in the same manner as described for Experiment I. For the degraded group, a grid of black lines was superimposed over the entire viewing screen so that the letters were viewed behind the grid. The lines on the grid, with a thickness of $.1^{\circ}$ of visual angle, were arranged with $.75^{\circ}$ of visual angle between adjacent horizontal and vertical lines. Half of the subjects in each group again indicated Same responses with the right hand and Different response with the left hand, while the remaining subjects had the opposite arrangement.

Subjects. Twelve men and 12 women from the same population as Experiment I were randomly assigned to the experimental conditions until it became necessary to balance for sex.

\section{Results and Discussion}

Errors. The error rate differences between center and peripheral visual field trials were similar to those reported for Experiment I and will not be reviewed in detail. However, on the peripheral visual field trials, the overall error rate was larger than for the first day of Experiment I, 16.1\% overall-9.9\% for intact stimuli and $22.2 \%$ for degraded stimuli-a significant main effect of stimulus quality, $F(1,22)=$ 25.203, $\mathrm{p}<.001$. As in Experiment $\mathrm{I}$, there were many more errors on Same trials $(25 \%)$ than on Different trials $(7.3 \%), F(1,22)=50.205, p<.001$. Consequently, separate analyses were conducted for Same and Different pairs.

The left panel of Figure 2 shows the percentage of errors on Same trials as a function of 90-trial blocks for LVF-RH and RVF-LH trials in both the intact (lower curves) and degraded (upper curves) stimulus presentation conditions. For the intact stimuli, there was again a shift away from an initial LVF-RH advantage, replicating the corresponding results from Experiment I. However, the pattern of results was much different for the degraded stimuli, for which the RVF-LH trials showed no improvement at all over the three trial blocks. These different patterns for intact vs. degraded stimuli produced a significant Linear Trial Block by Visual Field by Stimulus Quality interaction, $F(1,22)=5.356, p<.05$, and, averaged over trial blocks, a significant Visual Field by Stimulus Quality interaction, $F(1,22)=5.282$, $\mathrm{p}<.05$. Averaged over all trial blocks, there was a highly significant LVF-RH advantage for the degraded stimuli, $F(1,11)=18.132, p<.005$, while there was no overall visual field difference for the intact stimuli, $F<1$. There were no significant error rate effects involving visual field for either intact or degraded different-name letter pairs.

Reaction time. Median RTs of correct responses were computed for each subject in the same manner as for Experiment I. Attention will be restricted to the left and right visual field trials because differences between center and peripheral visual field trials, including the Visual Field by Same-Different interaction, were very similar to those reported for Experiment I. It should be noted at the outset that RT on peripheral field trials in the present experiment must be interpreted somewhat cautiously, especially for the degraded stimuli, because of the higher error rates. Nevertheless, it is instructive to examine the theoretically relevant $\mathrm{RT}$ differences.

Averaged over all other experimental conditions, RT was significantly faster for intact stimuli $(869 \mathrm{msec})$ than for degraded stimuli $(1146 \mathrm{msec})$, $\mathrm{F}(1,22)=15.549, \mathrm{p}<.001$. In addition, there was a significant Stimulus Quality by Same-Different interaction, $F(1,22)=14.041, \mathrm{p}<.005$, as the

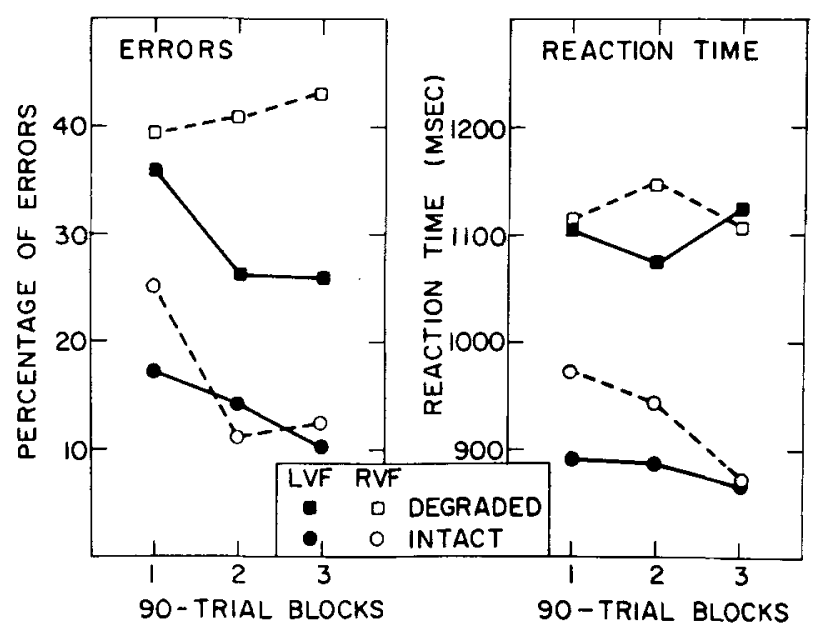

Figure 2. Percentage of errors (left panel) and mean $\mathrm{RT}$ in milliseconds (right panel) for same-name letter pairs as a function of 90-trial blocks. Each panel presents data for left (LVF) and right (RVF) visual field trials for both intact and degraded stimuli. 
stimulus quality RT difference was larger for different-name pairs than for same-name pairs.

The right panel of Figure 2 shows RT in milliseconds to Same pairs as a function of 90-trial blocks for LVF-RH and RVF-LH trials in both the intact (lower curves) and degraded (upper curves) stimulus presentation conditions. As shown in Figure 2, for the intact stimuli there was a larger decrease in RT over trial blocks for RVF-LH trials than for LVF-RH trials, resulting in a shift in RT advantage away from the initial LVF-RH advantage. This replicated the corresponding Day 1 effect from Experiment $I$ and produced a significant Linear Trial Block by Visual Field interaction for samename letter pairs, $F(1,11)=5.639, p<.05$. As in Experiment I, 10 of 12 subjects showed a shift in this direction. Note, however, that even on the third trial block in the present experiment there was no RVF-LH advantage. This difference from Experiment I may be attributable to slightly different viewing conditions, to the different group of individuals, or to some other factor. Regardless of the reason for this discrepancy, it appears that the systematic shift in relative RT advantage that occurs as a function of practice is a more stable phenomenon than the absolute direction of RT advantage at any one stage of practice.

The RT pattern shown in Figure 2 for the samename degraded stimuli was quite different. When the perceptual difficulty was maintained at a high level, there were no systematic decreases in RT over trial blocks for either RVF-LH or LVF-RH trials. Consequently, the Linear Trial Block by Visual Field interaction that had been obtained on Day 1 of Experiment I and for intact stimuli in the present experiment did not approach statistical significance for the degraded stimuli, $F<1$. None of the effects involving visual fields approached significance for either intact or degraded different-name letter pairs.

\section{GENERAL DISCUSSION}

The present experiments suggest that two general processing stages are critical for determining the type of laterality effects obtained for judgments of whether two different-case letters have the same name: a visuospatial processing stage where information about such things as visual features must be extracted and a more abstract, verbal stage where letter names are attached to the perceptually recognized features and the letter names are compared. The results further suggest that the right hemisphere is more specialized than the left for the visuospatial stage of letter processing while the left hemisphere is more specialized than the right for the verbal stages of analysis (cf. Hellige \& Cox, 1976; Kimura, 1973). The laterality pattern obtained appears to be determined, at least in part, by the relative difficulty of the two general processing stages. Therefore, even a task that demands some verbalization may lead to a sizeable LVF-RH advantage if the visuospatial aspects of the task are made very difficult. Even when no systematic attempt is made to perceptually degrade the stimuli, the difficulty of the visuospatial processing would seem to be greater at the beginning of these types of visual perception tasks than after considerable practice. Therefore, the laterality patterns would change over the early stages of practice in the manner reported for intact stimuli in Experiments I and II because of decreases in the difficulty of visuospatial stage of information processing.

At no level of practice in either experiment were there any RVF vs. LVF error rate or RT differences for different-name letter pairs. As noted in the introduction, the absence of any RVF-LH advantage for different-name letter pairs has been reported by several previous investigators using this paradigm, even when such an advantage is reported for samename pairs. For foveally presented letter and form pairs, RTs are faster for Same pairs than for Different pairs, but this difference is eliminated or reversed on peripheral visual field trials (Experiments I and II; Hellige, 1975; Lefton \& Haber, 1974). Based on these results, Lefton and Haber suggest that subjects are relatively biased toward sameness for foveal stimuli and relatively biased toward difference for peripheral stimuli. The larger error rates on Same trials than on Different trials for peripheral stimuli in the present experiments are consistent with this response bias hypothesis. Therefore, on peripheral trials, correct responses to Same pairs may reflect the extraction of more visual and verbal information than do correct responses to Different pairs. Stated another way, more correct Different responses are likely to be guesses based on insufficient information. Consequently, performance on Same trials may be a better indication of the psychological processes involved in extracting sufficient information to make a correct decision, so that any hemispheric differences in these processes would appear larger for Same pairs than for Different pairs. Although speculative, this hypothesis could be more completely examined by systematically manipulating Same-Different response biases, and is consistent with the results of the present experiment and others discussed earlier.

\section{Conclusions}

The present experiments resulted from attempts to explain discrepancies among recent visual laterality experiments which employ the same basic paradigm, a necessary step if laterality patterns are to reveal anything about hemispheric specialization. The combined results of the present experiments make explicit the effect on laterality patterns of several related variables that could cause such discrepancies: level of practice, perceptual quality of visual stimuli, 
and possible same-different response biases. These results have implications beyond the specific manipulations reported here because other variables that change stimulus quality or response biases would also be expected to influence laterality patterns. The experiments reported here indicate that in order to more fully understand laterality patterns it is necessary to take into account all of the stages of information processing that are required to perform an experimental task and determine relative hemispheric specialization for each of these processing stages.

\section{REFERENCES}

CoHen, G. Hemispheric differences in a letter classification task. Perception \& Psychophysics, 1972, 11, 139-142.

Davis, R., \& Schmit, V. Visual and verbal coding in the interhemispheric transfer of information. Acta Psychologia, 1973, 37, 229-240.

Geffen, G., Bradshaw, J. L., \& Netrteton, N. C. Hemispheric asymmetry: Verbal and spatial encoding of visual stimuli. Journal of Experimental Psychology. 1972, 95, 25-31.

Hellige, J. B. Hemispheric processing differences revealed by differential conditioning and reaction time performance. Journal of Experimental Psychology: General, 1975, 104, 309-326.

Hellige, J. B., \& Cox, P. J. Effects of concurrent verbal memory on recognition of stimuli from the left and right visual fields. Journal of Experimental Psychology: Human Perception and Pertormance, 1976, 2, 210-221.

Kallman, H. J., \& Corballis, M. C. Ear asymmetry in reaction time to musical sounds. Perception \& Psychophysics, 1975, 17, 358-370.
Kimura, D. The functional asymmetry of the brain in visual perception. Neuropsychologia, 1966, 4, 275-285.

KimurA, D. The asymmetry of the human brain. Scientific American, 1973, 228, 70-78.

Kimura, D., \& Durnford, M. Normal studies on the function of the right hemisphere in vision. In S. J. Dimond \& J. G. Beaumont (Eds.). Hemisphere function in the human brain. New York: Wiley, 1974. Pp. 25-47.

Lefton, L. A., \& Haber, R. N. Information extraction from different retinal locations. Journal of Experimental Psychology, $1974,102,975-980$.

STERnBERG, $S$. The discovery of processing stages: Extensions of Donder's method. In W. G. Koster (Ed.), Attention and performance II. Acta Psychologica, 1969, 30, 276-315.

Sternberg, S. Memory scanning: New findings and current controversies. Quarterly Joumal of Experimental Psychology, 1975, 27, 1-32.

VANDERPlas, J. M., \& Garvin, E. A. The association values of random shapes. Journal of Experimental Psychology, 1959, 57, 147-154.

Wilkins, A., \& Stewart, A. The time course of lateral asymmetries in visual perception of letters. Journal of Experimental Psychology, 1974, 102, 905-908.

\section{NOTE}

1. A certain amount of caution must be exercised in interpreting trend analyses when the time between successive blocks of trials is not constant (e.g., within day vs. between days). Nevertheless, such analyses do serve to clarify the manner in which RT decreased with practice in the present experiment.

(Received for publication, April 9, 1976; revision accepted, July $16,1976$. ) 